\title{
Clinical Features of Oxaliplatin Induced Hypersensitivity Reactions and Therapeutic Approaches
}

\author{
Nusrat Bano ${ }^{1 *}$, Rahila Najam² ${ }^{2}$ Faaiza Qazi ${ }^{3}$, Ahmed Mateen $^{4}$
}

\begin{abstract}
Oxaliplatin, a third generation novel platinum compound is the most effective first line chemotherapeutic agent for colorectal cancer (CRC) in combination with 5FU and leucovorin. It is indicated for pancreatic, gastric and testicular cancers combined with bevacuzimab, capecitabine, irinotecan and other cytotoxic agents. However, moderate to severe hypersensitivity reactions (HSR) during or after oxaliplatin infusion usually require cessation of chemotherapy or substitution of the key therapeutic drug which largely interferes with improved patient prognosis. This mini- review showcases recent and accepted opinions/approaches in oxaliplatin induced HSR management. Physicians and oncologists have varying attitudes regarding the decision to rechallenge the patient after an HSR experience, efficacy of desensitization protocols, effectiveness and selection of drugs for premedication and possibilities of cross sensitivity to other platinum agents (e.g. carboplatin). A brief insight into underlying molecular mechanisms and clinical manifestations of oxaliplatin induced HSR is offered. We have also discussed the management of oxaliplatin induced HSR and risk stratification for a successful and complete chemotherapeutic plan.
\end{abstract}

Keywords: Oxaliplatin - hypersensitivity reactions - desensitization - management - rechallenge

Asian Pac J Cancer Prev, 17 (4), 1637-1641

\section{Introduction}

Oxaliplatin [trans-L-dach $(1 \mathrm{R}, 2 \mathrm{R}$-diaminocyclo hexane) oxalatoplatinum, L-OHP] is one of the mainstay chemotherapeutic drugs in gastrointestinal cancers and may cause hypersensitive reactions with fatal outcomes (Aroldi et al., 2015). Hypersensitivity reactions are reported in $12 \%$ of patients treated with oxaliplatin, whereas $1 \%$ of these patients may face a life threatening situation. Extended steroid premedication with slower oxaliplatin infusion can be employed for safety in patients after severe hypersensitivity reaction with oxaliplatin (Nisi et al., 2015). Coinfusion of dexamethasone with oxaliplatin is considered to effectively reduce oxaliplatin induced hypersensitivity reactions (Yoshida et al., 2015). Although desensitization of platinum compounds (carboplatin) is associated with poor efficacy (Dodgshun et al., 2016), the effectiveness of oxaliplatin desensitization is implied to be effective (Syrigou, 2015). Better therapeutic care with oxaliplatin may be achieved by desensitization protocol focusing on oxaliplatin specific immunoglobin E (Madrigal et al., 2013). The optimum oxaliplatin desensitization protocol is yet to be defined despite efficacy and tolerability of existing protocols (Okayama et al., 2015). On the other hand, false negative sensitivity reactions to oxaliplatin can be out ruled by risk-stratification protocol thus limiting unnecessary desensitization and better therapeutic effects (Wang et al., 2015).

Drug provocation tests in diagnostic protocols are shown to effectively indicate oxaliplatin hypersensitivity in susceptible patients and avoid unnecessary desensitization (Alvarez et al., 2015). Difficult breakthrough reactions are an added concern in patients with remote drug exposure during oxaliplatin desensitization (Kim et al., 2015). Although the risk of hypersensitivity reaction exists with each infusion of chemotherapeutic agent (Lundqvist et al., 2012), it is higher in heavily pretreated patients (Wang et al., 2012). Risk of hypersensitivity reaction to oxaliplatin (12-15\%) usually exists after the sixth cycle of treatment (Lee et al., 2013). Chances of hypersensitivity reactions after desensitization of oxaliplatin lie in the initial 2-3 cycles of treatment (Pedersen, 2015).

\section{Incidence Rate and Dose Intensity}

Incidence rate of Type 1 anaphylactic reactions with platinum analogues like carboplatin and cisplatin is 5\%, compared to which fewer allergic reactions attributed to oxaliplatin are reported in allergenic studies (Larzilliere et al., 1999; Alliot et al., 2001; Arotcarena et al., 2001). There are earlier reports of anaphylaxis during $6^{\text {th }}$ cycle 
of chemotherapy in one out of 46 patient receiving a dose of $100 \mathrm{mg} / \mathrm{m}^{2}$ oxaliplatin every $2^{\text {nd }}$ week (de Gramount et al., 1997). The propensity of allergic reactions with platinum compounds lies with the increased number of cycles during chemotherapy (Shlebak et al., 1995; Polyzos et al., 2001). High incidence rate of allergic reactions $(27 \%)$ is reported by Markman et al. (1999) in patients who received more than seven cycles of treatment.

Incidence rate of oxaliplatin based hypersensitivity reactions is higher in second line treatments with palliative concern (19.6-24\%) compared to adjuvant chemotherapy (9-10\%) particularly when the first regimen is based on irinotecan (Caiado and castells, 2015). Gerard et al. (1998) reported symptoms of allergy in one out of 37 patients in 8th cycle of treatment with $130 \mathrm{mg} / \mathrm{m}^{2}$ oxaliplatin (2hrs infusion) every three week despite prophylactic pretreatment with corticosteroids. Severe anaphylaxis in two patients out of sixty (receiving dose of $100 \mathrm{mg} /$ $\mathrm{m} 2$ every 2 weeks) is also reported during 8 and 9 cycle of treatment (Maindrault et al., 1999). Thomas et al. (2003) reported three cases of allergic reactions during a phase 1 trial of oxaliplatin (given D1 once 3 weekly) with capecitabine, although all of the patients received dexamethasone with ondasetron as pretreatment. A recent retrospective study reports HSR incidence rate $(11.3 \%)$ at average total dose of oxaliplatin of $622.2 \mathrm{mg} /$ $\mathrm{m}^{2}$ (Yamauchi et al., 2015). Higher incidence rate of hypersensitivity reactions is associated with female gender and younger age (Parel et al., 2014).

\section{Underlying Mechanisms of Oxaliplatin Induced HSR}

Essentially unpredictable, patient specific and individual predisposing factors lead to hypersensitivity drug reactions (Brockow et al., 2015). Allergic reactions to platinum compounds are associated with platinum sensitive IgE antibody formation, after platinum inhalation characterized by urticaria and dyspnea (Leguy- Seguin et al., 2007). There can be a subsequent rise in serum levels of TNF (tumor necrosis factor) and Interleukin during the allergic episode which attained normal values when the allergy had subsided (Santini et al., 2001). Oxaliplatin induced allergic reactions are classified and distinguished as Hypersensitivity adverse effects or infusional idiosyncratic reactions (Thomas et al., 2003). Hypersensitivity or anaphylactic reaction by oxaliplatin is due to antigen to antibody (mast cell fixed, cytophilic (IgE) antibodies) reaction and causes smooth muscle contractions with capillary dilation, due to the release of mediators of inflammation from the mast cells rupture.

The second category of adverse reaction with oxaliplatin presenting allergy like symptoms are infusional or idiosyncratic reactions. These reactions are abnormal but they are not antibody dependent. They are usually delayed. In few such case reports (Santini et al., 2001; Tonini et al., 2002), hypotension was not associated with the symptoms of allergy (i.e. fever, chills, diarrhea, abdominal pain). The abnormal feature marked in these cases was no fall in blood pressure but a rise in TNF and IL-6 noted during the reactionary phase. In cases of
Evans syndrome or hemolytic anemia associated with oxaliplatin, positive DAT results are reported for IgG or C3 complement proteins (Cobo et al., 2007). Decreased levels of ADAMTS13 were found in oxaliplatin-induced thrombotic thrombocytopenic purpura without specific antibodies to this protein (Baretta et al., 2013; Malkhasyan et al., 2015). Fibrinolysis induced by oxaliplatin may be the underlying mechanism of disseminated intravascular coagulation (DIC) (kurian et al., 2012). In a more recent case report of DIC, Malkhasyan et al. (2015) suggested that reactive oxaliplatin-dependent autoantibodies caused acute intravascular hemolysis which later on complicated DIC. Oxaliplatin-dependent antiplatelet antibodies or antibodies against GP IIIa may also have a role in DIC (Malkhasyan et al., 2015; Spearing et al., 1990). Adsorption of oxaliplatin on blood cells may result in the formation of autoantibodies to platelets and erythrocytes that lead to oxaliplatin induced hemolytic anemia and thrombocytopenia (Taleghani et al., 2005; Curtis et al., 2006; Caiado et al., 2015).

\section{Clinical Manifestatons of Oxaliplatin Induced HSR}

Presentation of unusual signs and symptoms during or after oxaliplatin infusion such as rigors, chills, fever, abdominal or back pain, hematuria, dark urine, ecchymosis, hematochezia, hematemesis, epistaxis, petechiae, or altered mental status require prompt complete blood test and evaluation (Forcello et al., 2015). Hypersensitivity reactions to oxaliplatin can give way to oxaliplatin induced thrombocytopenia. A recent study reports Grade 1 erythema in $4.1 \%$ patients (Yoshinda et al., 2015). Hypersensitivity reactions with oxaliplatin are manifested as urticaria, rash, itching flushing, flare, edema, dyspnea, laryngoedema, bronchospasm, diaphoresis, disorientation and syncope (Cheng et al., 2008). Machover et al (1996) reported a case of transient dyspnea in 1 out of 109 patients receiving $130 \mathrm{mg} / \mathrm{m} 2$ every three weeks. Few cases of HSR with oxaliplatin presented with urticaria and dyspnea are reported in a recent study (Pagani, 2015). FOLFOX (an oxaliplatin based regimen) is effective and considerably safe chemotherapeutic regimens in colorectal carcinoma (CRC) (Bano et al., 2014; Toki et al., 2014). Florit-Sureda et al. (2015) reported a case of HSR in a 56 year old male presented with pruritis, flushing, erythema, abdominal pain and oedema over face and thorax after 15 minutes of initiating infusion of oxaliplatin and folinic acid on the tenth cycle of FOLFOX 6. Tamura (2015) reported a case of anaphylactic shock leading to cardiopulmonary arrest. Allergic reaction with fever after oxaliplatin infusion is reported (Yanagihara, 2015).

A retrospective study has shown that thrombocytopenia develops in $7.1 \%$ of patients with allergic manifestations to oxaliplatin (Maindrault et al., 1999). Osumi et al. (2013) reported three cases of fatal thrombocytopenia after oxaliplatin chemotherapy. Tournigand (1998) reported a case of immediate allergic reaction in a patient who had received oxaliplatin infusion over 2-3 hrs, with the symptoms of flushing sweating, redness of face and neck associated with hypotension after initiation of $7^{\text {th }}$ cycle of 
oxaliplatin at a dose of $100 \mathrm{mg} / \mathrm{m}^{2}$. The symptoms resolved after 9 hrs after oxygen, volume expansion, epinephrine and dexamethasone. The patient was subjected to chemotherapy after 2 weeks, allowing a slower infusion rate but the symptoms of allergic reactions returned and required discontinuation of therapy. The study reported four other patients who experienced a similar pattern of allergy due to oxaliplatin treatment and discontinuation of treatment. HSRs were reported by Meyer et al. (2002) in eight patients who experienced mild symptoms of allergic reaction. Santini et al. (2001) reported two episodes of oxaliplatin hypersensitivity reactions with vomiting, chills and fever associated with abdominal pain and diarrhea. Whikle these resolved in the next cycle with prednisone pretreatment, allergic symptoms returned in the following cycle when the patient did not receive prednisone.

A case of systemic capillary leak syndrome is reported for the first time with oxaliplatin chemotherapy in a 63 year old male patient during $12^{\text {th }}$ cycle of infusion (Anderson, 2015). Acute onset of fulminant disseminated intravascular coagulation with acute hemolytic anemia during oxaliplatin chemotherapy infusion is reported in a 61 year old male patient (Malkhasyan et al., 2015). Interstitial lung disease induced by single agent oxaliplatin as a less common adverse reaction is reported by Kumaran et al. (2015). Other than type II hypersensitive reactions (hemolytic anemia and thrombocytopenia), oxaliplatin is also associated with delayed hypersensitive reactions (De Vries et al., 2006; Tham et al., 2015)

\section{To Rechallenge the Patient or Not}

Temporary interruptions of the infusion can resolve mild to moderate allergic reactions and may further require reduction in rate of infusion with symptomatic management, however rechallenging the patient can be considered as an option only after the symptoms have completely resolved (Lenz, 2007). Thomas et al. (2003) advocate with literature reports backup that the patients who develop mild to moderate allergic and infusional reactions can be rechallenged safely after pretreatment with steroids and histamine receptor blockers. Ondasetron as an antiemetic pretreatment drug may however be replaced by metochlopramide as it has a tendency to elucidate allergy. However the patients with severe allergic manifestations should not be rechallenged to oxaliplatin exposure even is if appropriate symptomatic management is given/planned (Polyzos, 2001). Ichikawa et al. (2009) reported recurrence of allergic reactions within 3 cycles of chemotherapy when the patients were rechallenged. Some studies report that patients who experienced allergy with carboplatin were successfully shifted to oxaliplatin (Shukunami et al., 1999; Zanotti et al., 2001; Gutierrez et al., 2002). On the contrary, there are case reports when patients prone to allergy with carboplatin experience cross reactivity with oxaliplatin (Shlebak et al., 1995; Polyzos et al., 2001).

\section{Management of Oxaliplatin Induced HSR}

In hypersensitivity reactions during platinum infusion antigen-stimulated mast cells release prostaglandins, leukotrienes, histamine and other factors (Bahl and Dean, 2015). Premedication, skin testing and desensitization protocols are employed for continuation of chemotherapy in patients who have had an allergic reaction to the drug but the treatment options for the disease are limited (Boulanger et al., 2014).

On the other hand severity of oxaliplatin induced HSR may require cessation of chemotherapy (Benedik et al., 2015). Rapid drug desensitization is considered to be the only effective procedure for overcoming HSR to first-line chemotherapy (Giavina et al., 2015). In severe chemotherapy induced anaphylactic reactions, acute management ensues with epinephrine (Intramuscular), supplemental oxygen, fluid resuscitation, steroids and bronchodilators. Premedication with antihistamines (H1 blocker-diphenhydramine $50 \mathrm{mg} \mathrm{IV}, \mathrm{H} 2$ blocker-ranitidine $50 \mathrm{mg}$ IV) before chemotherapeutic infusion is required to ameliorate and attenuate risk of allergic reactions (Tham et al., 2015).

Coinfusion of oxaliplatin and dexamethasone followed by premedication with steroids and palonosetron is also reported to effectively reduce the incidences of oxaliplatin induced HSR (Yoshinda et al., 2015). Monteleukast and ASA has rendered effective therapeutic solution to reduce the risk and severity of platinum induced HSR (Breslow et al., 2009). Cheng et al. (2008) reported 25 (11 females and 14 males) cases of allergic reactions in a prospective study in CRC patients treated with FOLFOX 4. Grade 1 allergy was reported in thirteen patients; grade 2 allergy in eight patients and grade 3 allergic reactions in 4 patients. On the onset of allergic reactions, oxaliplatin infusion was interrupted and substituted with fluid support. Symptomatic management with corticosteroids, histamine receptor blockers and oxygen led to alleviation of the symptoms. The anaphylactic reaction in these cases was sufficiently contained and none of the patients required intensive monitoring and no case of mortality was reported. The study reported $76 \%$ (19 of 25 patients) success rate of completion of oxaliplatin based chemotherapy in the patients despite the onset of allergic reactions which was effectively tackled with symptomatic management. Prolongation of oxaliplatin infusion time of is an effective measure for management of the allergic reactions (Schull et al., 2001; Cheng et al., 2008).

\section{Conclusions}

Oxaliplatin induced HSR is presented with clinical signs of both acute and delayed allergies ranging in severity and require close monitoring for accurate diagnosis and quantification of dose. Although the standard of therapeutic care can be attained by rapid desensitization, risk of hypersensitivity reactions to oxaliplatin can be reduced by the effective use of premedication to stabilize the mast cells and promptly block receptors for the mediators of inflammation. Prolongation of infusion time is an effective strategy to cope with allergenic tendency of oxaliplatin in susceptible patients. Cross sensitivity to carboplatin cannot be entirely neglected. Longer platinum free survival adds to the risk of HSR. 


\section{References}

Aroldi F, Prochilo T, Bertocchi P, et al (2015). Oxaliplatininduced hypersensitivity reaction: underlying mechanisms and management. J Chemother, 27, 63-6.

Anderson BJ, Peterson LL (2015). Systemic capillary leak syndrome in a patient receiving adjuvant oxaliplatin for locally advanced colon cancer. J Oncol Pharm Pract, 12, 1078155215591388.

Alvarez-Cuesta E, Madrigal-Burgaleta R, Angel-Pereira D, et al (2015). Delving into cornerstones of hypersensitivity to antineoplastic and biological agents: value of diagnostic tools prior to desensitization. Allergy, 70, 784-94.

Arotcarena R, Berthelemy P,Piot M, et al (2001). Readministration of oxaliplatin using a rapid desensitization method after severe anaphylactic reaction. Gastroenterol Clin Biol, 25, 206-7.

Alliot C, Messouak D, Beets C, et al (2001). Severe anaphylactic reaction to oxaliplatin. Clin Oncol ( $R$ Coll Radiol), 13, 236.

Benedik J (2015). Hypersensitivity: case report. Reactions, 1534, 137.

Bahl M, Dean T (2015). An intermediate step for the management of hypersensitivity to platinum and taxane chemotherapy. Curr Oncol, 22, 220.

Brockow K, Przybilla B, Aberer W, et al (2015). Guideline for the diagnosis of drug hypersensitivity reactions. Allergo $J$ Int, 24, 94-105.

Bano N, Najam R, Qazi F, et al (2014). Gastrointestinal Adverse Effects in Advanced Colorectal Carcinoma Patients Treated with Different Schedules of FOLFOX. Asian Pac J Cancer Prev, 15, 8089-93.

Boulanger J, Boursiquot JN, Cournoyer G, et al (2014). Comite de l'evolution des pratiques en oncologie. Curr Oncol, 21, 630-41.

Baretta Z, Falci C, Piva E et al (2013). Fatal oxaliplatin-induced thrombotic thrombocytopenic purpura: a case report. Clin Colorectal Cancer, 4, 294-96.

Breslow RG, Caiado J, Castells MC (2009). Acetylsalicylic acid and montelukast block mast cell mediator-related symptoms during rapid desensitization. Ann Allergy Asthma Immunol, 102, 155-60.

Caiado J, Castells M (2015). Presentation and diagnosis of hypersensitivity to platinum drugs. Curr Allergy Asthma Rep, 15, 1-9.

Cheng LC, Chen HH, Lin SE, et al (2008). Hypersensitivity reactions to oxaliplatin: a prospectively collected study of 25 cases treated in one institute. J Soc Colon Rectal Surgeon (Taiwan), 19, 115-21.

Cobo F, De Celis G, Pereira A, et al (2007). Oxaliplatin-induced immune hemolytic anemia: a case report and review of the literature. Anti-Cancer Drugs, 18, 973-6.

Curtis BR, Kaliszewski J, Marques MB, et al (2006). Immunemediated thrombocytopenia resulting from sensitivity to oxaliplatin. Am J Hematol, 81, 193-8.

Dodgshun AJ, Hansford JR, Cole T, et al (2016). Carboplatin hypersensitivity reactions in pediatric low grade glioma are protocol specific and desensitization shows poor efficacy. Pediatr Blood Cancer, 63, 17-20.

De Vries RS, Mattijssen EJ, van Sorge AA, (2006). Serious delayed hypersensitivity reaction to oxaliplatin. Ann oncol, 17, 1723-4.

De Gramont A, Vignoud J, Tournigand C, et al (1997). Oxaliplatin with high-dose leucovorin and 5-fluorouracil 48-hours continuous infusion in pre-treated metastatic colorectal cancer. Eur J Cancer, 33, 214-9.

Florit-Sureda M, Conde-Estevez D, Vidal J, et al (2015). Hypersensitivity reaction caused by folinic acid administration: a case report and literature review. $J$ Chemother. [Epub ahead of print]

Forcello NP, Khubchandani S, Patel SJ, et al (2015). Oxaliplatininduced immune-mediated cytopenias: A case report and literature review. J Oncol Pharm Pract, 21, 148-56.

Giavina-Bianchi P, Aun MV, Galvao VR, et al (2015). Rapid Desensitization in Immediate Hypersensitivity Reaction to Drugs. Curr Treat Options Allergy, 2, 268-85.

Gutierrez M, Pautier P, Lhomme C, et al (2002). Replacement of carboplatin by oxaliplatin may be one solution for patients treated for ovarian carcinoma who are hypersensitive to carboplatin. J Clin Oncol, 20, 353.

Gerard B, Bleiberg H, Van Daele D, et al (1998). Oxaliplatin combined to 5-fluorouracil and folinic acid: an effective therapy in patients with advanced colorectal cancer. Anticancer Drugs, 9, 301-05.

Ichikawa Y, Goto A, Hirokawa S, et al (2009). Allergic reactions to oxaliplatin in a single institute in Japan. Jpn J Clin Oncol, 39, 616-20.

Kim JY, Lim KH, Song WJ, et al (2015). Breakthrough Reactions during Oxaliplatin Desensitization: An Analysis of 177 Cases. J Allergy Clin Immunol, 135, 112.

Kumaran D, Rupa D, Haresh KP, et al (2015). Oxaliplatin induced pulmonary toxicity-a rare phenomenon. Cancer Treat Commun, 4, 203-5.

Kurian S, Macintyre J, Mushtaq M, et al (2012). Oxaliplatin induced disseminated intravascular coagulation: A case report and review of literature. World J Gastrointest Oncol, 4, 181 .

Lee SY, Kim MY, Kim MH, et al (2013). Hypersensitivity reactions to oxaliplatin: outcomes of premedication and desensitization. J Allergy Clin Immunol, 131, 166.

Lundqvist EÅ, Fujiwara K, Seoud M (2015). Principles of chemotherapy. Int J Gynaecol Obstet, 131, 146-9.

Leguy-Seguin V, Jolimoy G, Coudert B, et al (2007). Diagnostic and predictive value of skin testing in platinum salt hypersensitivity, J Allergy Clin Immunol, 119, 726-30.

Lenz HJ (2007). Management and preparedness for infusion and hypersensitivity reactions. Oncologist, 12, 601-9.

Larzilliere I, Brandissou S, Breton P, et al (1999). Anaphylactic reaction to oxaliplatin: a case report. Am J Gastroenterol, 94, 3387-8.

Malkhasyan K, Halene S, Lacy J, et al (2015). OxaliplatinRelated Acute Disseminated Intravascular Coagulation Syndrome in a Patient With Metastatic Colon Cancer. Clin Colorectal Cancer, 14, 9-12.

Madrigal-Burgaleta R, Berges-Gimeno MP, Angel-Pereira D, et al (2013). Hypersensitivity and desensitization to antineoplastic agents: outcomes of 189 procedures with a new short protocol and novel diagnostic tools assessment. Allergy, 68, 853-61.

Meyer L, Zuberbier T, Worm M, et al (2002). Hypersensitivity reactions to oxaliplatin: cross-reactivity to carboplatin and the introduction of a desensitization schedule [letter]. J Clin Oncol, 20, 1146-7.

Markman M, Kennedy A, Webster K, et al (1999). Clinical features of hypersensitivity reactions to carboplatin. J Clin Oncol, 17, 1141-5.

Maindrault-Goebel F, Louvet C, Andre T, et al (1999). Oxaliplatin added to the simplified bimonthly leucovorin and 5-fluorouracil regimen as second-line therapy for metastatic colorectal cancer (FOLFOX6). GERCOR. Eur J Cancer, 35, 1338-42.

Machover D, Diaz-Rubio E, de Gramont A, et al (1999). Two consecutive phase II studies of oxaliplatin (L-OHP) for treatment of patients with advanced colorectal carcinoma who were resistant to previous treatment with 
fluoropyrimidines. Ann Oncol, 7, 95-8.

Nisi C, Moretti A, Donati D, et al (2015). E18A post-reaction regimen for CRC patients manifesting hypersensitivity to oxaliplatin: an effective alternative not to rule out an important option of treatment. Ann Oncol, 26, 41.

Okayama T, Ishikawa T, Sugatani K, et al (2015). Hypersensitivity reactions to oxaliplatin: identifying the risk factors and judging the efficacy of a desensitization protocol. Clin Ther, 37, 1259-69.

Osumi H, Shinozaki E, Kumekawa Y, et al (2013). Three cases of fatal thrombocytopenia after oxaliplatin-based chemotherapy. Gan Kagaku Ryoho, 40, 1557-60.

Pedersen KS (2015). Safety and efficacy of desensitization protocols for platinum hypersensitivity reactions. Pancreas, 2,10 .

Pagani M (2015). Hypersensitivity, urticaria and dyspnoea: 6 case reports. Reactions, 1567, 168-5.

Parel M, Ranchon F, Nosbaum A, (2014). Hypersensitivity to oxaliplatin: clinical features and risk factors. $B M C$ Pharmacol Toxicol, 15, 1.

Polyzos A, Tsavaris N, Kosmas C, et al (2001). Hypersensitivity reactions to carboplatin administration are common but not always severe: a 10-year experience. Oncol, 61, 129-33.

Syrigou EI (2015). Hypersensitivity reaction: case report. Reactions, 1533, 343-10.

Santini D, Tonini G, Salerno A, et al (2001). Idiosyncratic reaction after oxaliplatin infusion [letter]. Ann Oncol, 12, 132-3.

Schüll B, Kornek GV, Scheithauer W (2001). Idiosyncratic reaction after oxaliplatin: circumvention by use of a continuous infusional administration schedule. Ann Oncol. 12, 1653-4.

Shukunami K, Kurokawa T, Kubo M, et al (1999). Hypersensitivity reaction to carboplatin during treatment for ovarian cancer: successful resolution by replacement with cisplatin. Tumori, 85, 297-8.

Shlebak AA, Clark PI, Green JA (1995).Hypersensitivity and cross reactivity to cisplatin and analogues. Cancer Chemother Pharmacol, 35, 349-51.

Spearing RL, Hickton CM, Sizeland P, et al (1990). Quinineinduced disseminated intravascular coagulation. Lancet, 336, 1535-7.

Tham EH, Cheng YK, Tay MH, et al (2015). Evaluation and management of hypersensitivity reactions to chemotherapy agents. Postgrad Med J, 91, 145-50.

Tamura H (2015). Anaphylactic shock leading to cardiopulmonary arrest: case report. Reactions, 1552, 171-23.

Toki MI, Saif MW, Syrigos KN (2014). Hypersensitivity reactions associated with oxaliplatin and their clinical management. Expert Opin Drug Saf, 13, 1545-54.

Taleghani BM, Meyer O, Fontana S, et al (2005). Oxaliplatininduced immune pancytopenia. Transfusion, 45, 704-8.

Thomas RR, Quinn MG, Schuler B, et al (2003). Hypersensitivity and idiosyncratic reactions to oxaliplatin. Cancer, 97, 2301-7.

Tonini G, Santini D, Vincenzi B, et al (2002). Oxaliplatin may induce cytokine-release syndrome in colorectal cancer patients. J Biol Regul Homeost Agents, 16, 105-9.

Tournigand C, Maindrault-Goebel F, Louvet C, et al (1998). Severe anaphylactic reactions to oxaliplatin. Eur J Cancer, 34, 1297-8.

Wang AL, Patil SU, Long AA, et al (2015). Risk-stratification protocol for carboplatin and oxaliplatin hypersensitivity: repeat skin testing to identify drug allergy. Ann Allergy Asthma Immunol, 115, 422-8.

Wang JH, King TM, Chang MC, et al (2012). Oxaliplatin-induced severe anaphylactic reactions in metastatic colorectal cancer: case series analysis. World J Gastroenterol, 18, 5427-33.

Yamauchi H, Goto T, Takayoshi K, et al (2015). A retrospective analysis of the risk factors for allergic reactions induced by the administration of oxaliplatin. Eur J Cancer Care, 24, 111-6.

Yanagihara Y (2015). Allergic reaction and fever: 2 case reports. Reactions, 1555, 147-3.

Yoshida Y, Hirata K, Matsuoka H, et al (2015). A single-arm Phase II validation study of preventing oxaliplatin-induced hypersensitivity reactions by dexamethasone: the aVOiD trial. Drug Des Devel Ther, 9, 6067-73.

Zanotti KM, Rybicki LA, Kennedy AW, et al (2001). Carboplatin skin testing: a skin-testing protocol for predicting hypersensitivity to carboplatin chemotherapy. $J$ Clin Oncol, 19, 3126-9. 